\title{
ESTUDIO DEL CONTENIDO Y LA CALIDAD DE LA LIGNINA MEDIANTE PIRÓLISIS ANALÍTICA EN MADERA DE PINUS CARIBAEA.
}

\author{
CONTENT AND QUALITY STUDY OF THE LIGNIN BY ANALYTICAL \\ PYROLYSIS IN PINUS CARIBAEA
}

\author{
Esther Álvarez Godoy'; José Carlos de Carvalho Rodrigues ${ }^{2}$; \\ Ana Maria Martins Alves ${ }^{3}$; Daniel Álvarez Lazo ${ }^{4}$
}

\begin{abstract}
RESUMEN
En este trabajo se presentan los resultados alcanzados en la determinación del contenido de lignina así como de su calidad (relación H/G) en muestras de madera de Pinus caribaea var caribaea de tres localidades en la provincia de Pinar del Río, Cuba. El estudio se realizó en el Instituto de Investigación Científica Tropical de Lisboa, Portugal, en el marco del Proyecto GEMA. La técnica utilizada fue la Pirólisis analítica. Los resultados que se muestran representan el promedio de tres corridas experimentales y se obtuvieron valores cercanos al método Klason utilizado como referencia. Se encontró una correlación aceptable entre los contenidos del lignina-pirólisis y lignina Klason para esta especie, lo cual permite calcular el contenido de lignina Klason a través del contenido de ligninapirólisis. La pirólisis analítica es de gran utilidad en los programas de mejoramiento genético, en que es necesario el análisis de muchas muestras, ya que la introducción de parámetros como la composición química de la madera, induce al desarrollo de métodos expeditos de caracterización, reproducibles y de bajo costo, una vez que los métodos tradicionales son lentos, requieren mucha mano de obra y son de elevado costo.
\end{abstract}

Palabras claves: Pirólisis analítica, Pinus caribaea, lignina-pirólisis, lignina Klason, relación H/G.

\begin{abstract}
This work shows the lignin content determined as well as its quality ( $\mathrm{H} / \mathrm{G}$ ratio) in wood samples of Pinus caribaea var caribaea from three sites at Pinar del Río Province, Cuba. The study was carried out at the Tropical Scientific Investigation Institute, Lisbon, Portugal (Project GEMA). The Analytical pyrolysis was used as analytical method. The results represent the average of three repetitions and was obtained similar values to Klason method used as reference. An acceptable correlation was obtained between the Py-lignin contents and Klason lignin for this species. This may be possible to calculate the Klason lignin through the content Py-lignin content. The Analytic pyrolysis is of great utility in the programs of genetic improvement, in which it is necessary the analysis of many samples, since the introduction of parameters like the chemical composition of wood, induce to the development of expedite methods of characterization, reproducible and low cost, once the traditional methods are slow, require a lot of manpower and have a high cost.
\end{abstract}

Keywords: Analytical pyrolysis, Pinus caribaea, Py-lignin, Klason lignin, H/G ratio.

\footnotetext{
${ }^{1}$ Profesor. Centro de Estudios Forestales de la Universidad de Pinar del Río. Cuba. ${ }^{2}$ Investigador. Instituto de Investigación Científica Tropical. Lisboa. Portugal. ${ }^{3}$ Investigadora. Instituto Superior de Agronomía. Universidad Técnica de Lisboa. Portugal. ${ }^{4}$ Profesor. Centro de Estudios Forestales de la Universidad de Pinar del Río. Cuba. Autor para correspondencia: estheral@af.upr.edu.cu Recibido: 01 de diciembre 2006. Aceptado: 25 de junio 2007.
} 


\section{INTRODUCCION}

La creciente búsqueda de madera de elevada calidad, con menor variabilidad de sus propiedades y mayor rendimiento, coloca una gran presión al nivel del desarrollo sustentable del bosque, que pasa entre otros por el mejoramiento cualitativo del leño. En este sentido se desarrollan actualmente proyectos de mejoramiento genético, esencialmente orientados para la obtención de materia prima de mejor calidad para la industria de pasta para papel.

En estos programas de mejoramiento, en que es necesario el análisis de muchas muestras, la introducción de parámetros como la composición química de la madera induce al desarrollo de métodos expeditos de caracterización, reproducibles y de bajo costo, una vez que los métodos tradicionales son lentos, requieren mucha mano de obra y son de elevado costo (Rodrigues, 1998).

Las alteraciones, tanto de la estructura macroscópica, como de la composición química, pueden resultar de condiciones anormales de crecimiento, y pueden estar también asociadas a la formación de leño juvenil y adulto, de leño de inicio y de fin de estación, etc. (Rodrigues, 1998).

Las ligninas de las maderas de las resinosas (gimnospermas), de las frondosas (angiospermas, dicotiledóneas) y de las gramíneas (angiospermas, monocotiledóneas) difieren en su contenido en unidades precursoras - guaiacilo $(\mathrm{G})$, siringilo $(\mathrm{S})$ y p-hidroxifenilo $(\mathrm{H})$ (Fengel y Wegener, 1984; Pereira et al., 2003). A pesar de la variabilidad observada en la composición de las ligninas, las maderas de las resinosas, frondosas y gramíneas presentan, tendencialmente, ligninas de los géneros G, GS y HGS, respectivamente (Fengel y Wegener, 1984).

Poco se conoce sobre la variabilidad de la composición estructural de la lignina en términos de la relación H/G (p-hidroxifenilo/guaiacilo) en resinosas, y de pino en particular, y menos aún se sabe sobre el impacto de esta variabilidad en la producción de pastas para papel. Se sabe, no obstante, que las frondosas son más fáciles de deslignificar debido a la composición estructural de la lignina. El desconocimiento en relación a la composición estructural de la lignina resulta, en gran parte, del hecho de que los métodos químicos (húmedos) más frecuentemente usados para el análisis de la composición de la lignina (tioacidólisis y oxidación por permanganato), resultan más adecuados para el análisis estructural cualitativo que para el análisis cuantitativo.

Para estudios de este tipo se están aplicando técnicas modernas, no destructivas, como es el caso de la Pirólisis analítica. Sólo recientemente ha tenido algún desarrollo esta técnica para el análisis cuantitativo (Rodrigues et al, 1999; Kuroda et al, 2002). También es muy reciente que comienzan a compararse estos resultados obtenidos mediante pirólisis, con los métodos de referencia, particularmente con el contenido de lignina obtenido por pirólisis analítica y por el método Klason (Alves, 2004). Por otro lado, esta técnica puede ser empleada como referencia en el estudio de las ligninas cuando ellas son cuantificadas por otro método físico- químico como es la Espectroscopía Infrarroja (Alves et al, 2006).

Pirólisis es un término de origen griego que significa "descomponer por el calor" y consiste en quebrar térmicamente y en ausencia de oxígeno, los enlaces de macromoléculas, de modo que se obtengan moléculas de menor masa, que van a ser separadas por cromatografía gaseosa (GC) e identificadas por espectrometría de masa (MS). Normalmente, esto es conseguido por intermedio de un calentamiento rápido en una atmósfera inerte o al vacío (Galletti, 1995). Generalmente, la pirólisis se realiza a temperaturas mayores de $250-300^{\circ} \mathrm{C}$, más frecuentemente entre $500^{\circ} \mathrm{C} \mathrm{y} 800^{\circ} \mathrm{C}$. 
La pirólisis analítica presenta las siguientes ventajas (Meyer y Faix, 1992):

1. la cantidad reducida de muestra necesaria (aproximadamente $75 \mu \mathrm{g}$ );

2. la simplicidad de preparación de las muestras, una vez que sólo tienen que ser sometidas a secado y molida;

3. el reducido período de tiempo que el análisis requiere (de escasos minutos hasta una hora y treinta minutos);

4. la fácil identificación de los productos de la pirólisis, una vez que ya existen compilaciones disponibles.

Pinus caribaea var caribaea es la especie maderera más abundante en la provincia de Pinar del Río, mostrando diferencias en distribución y crecimiento para los distintos sitios de plantación. Para establecer un mejor manejo de la especie y la utilización de este recurso maderero con fines industriales, es importante conocer de la variación de las propiedades químicas, referidas a los contenidos de polisacáridos y lignina y la variación de estos según las localidades de procedencia.

\section{Objetivo}

Con el presente trabajo se pretende dar a conocer resultados novedosos en la aplicación de métodos no destructivos al estudio de la madera, particularmente la aplicación de la pirólisis analítica para la determinación del contenido y la calidad de la lignina de Pinus caribaea var caribaea en tres localidades de la provincia de Pinar del Río, Cuba.

\section{MATERIALES Y MÉTODOS}

Para el estudio se utilizaron muestras de madera de Pinus caribaea var caribaea, de plantaciones de 29 años de edad, provenientes de tres localidades de la provincia de Pinar del Río, Cuba:

Cajálbana, Marbajita y Malas aguas (figura 1). Fueron derribados 15 árboles, 5 para cada localidad. En cada tronco fueron separados discos con $5 \mathrm{~cm}$ de espesor a $1,30 \mathrm{~m}$ de altura $(5 \times 3=15$ muestras).

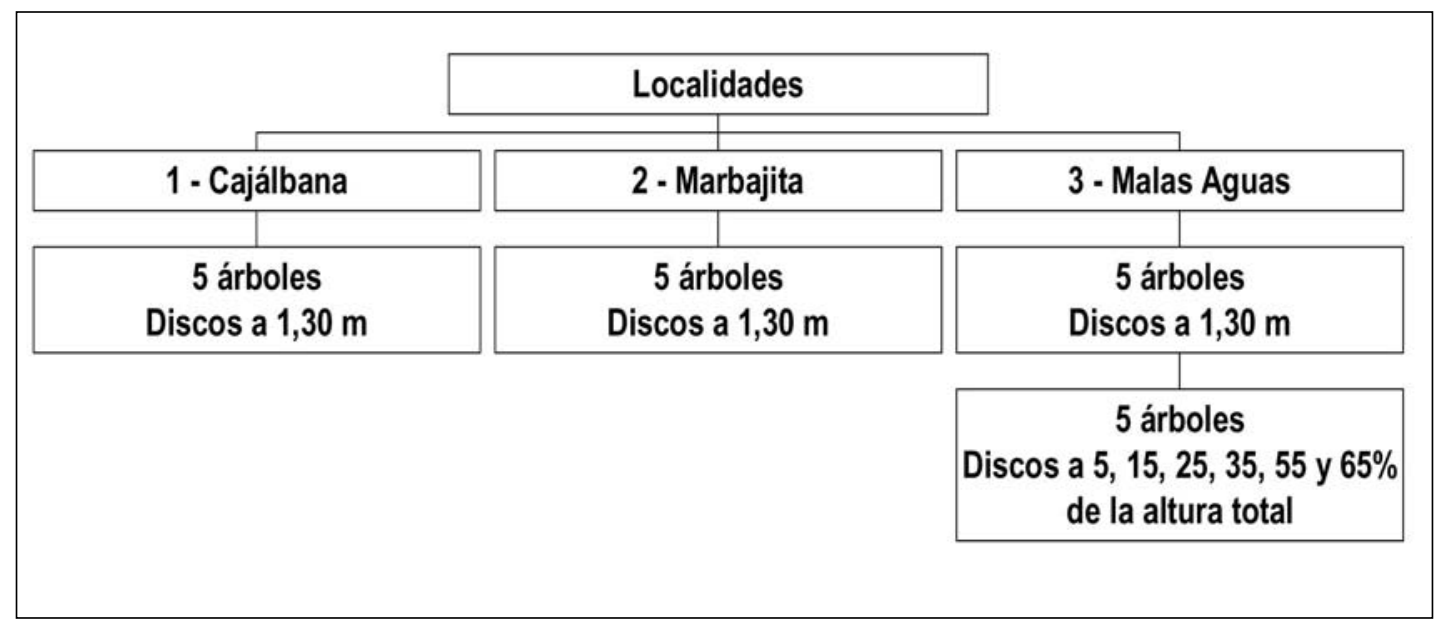

Figura 1:. Toma de las muestras 
Adicionalmente, para estudiar la variabilidad entre árboles y a diferentes alturas de un mismo árbol, se derribaron otros cinco árboles de la localidad de Marbajita y se obtuvieron discos a seis alturas del fuste, excepto en un árbol que originó solamente cinco discos $(5$ x $6=30 ; 30-1=29$ muestras).

Así se obtuvo un total de 44 muestras. $(15+29=44)$. De cada disco se retiró una pieza central, midiendo $2 \mathrm{~cm}$ a cada lado de la médula y fueron trasladadas al Instituto de Investigación Tropical de Lisboa. Las muestras fueron posteriormente cortadas como se muestra en la figura 2.

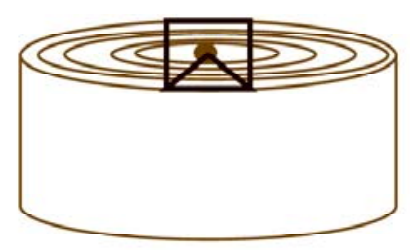

Figura 2:. Corte de las muestras

Una sección triangular se utilizó para el análisis químico. Las muestras fueron molidas en un molino de cuchillas Retsch modelo SM 2000 y posteriormente en un molino Thomas, obteniéndose partículas $<1 \mathrm{~mm}$.

La madera molida fue extraída en un sistema de tres disolventes con aumento de gradiente de polaridad (diclorometano, etanol, agua), en equipos de extracción Soxhlet. Después de la extracción se secaron las muestras en estufa durante aproximadamente 12 horas a $60^{\circ} \mathrm{C}$ y se mantuvieron en un desecador con pentóxido de difósforo al vacío hasta que volvieran a ser molidas, en molino ultracentrífuga de la marca Retsch ZM-100 con tamiz de malla $0,12 \mathrm{~mm}$, siendo nuevamente colocadas en un desecador con pentóxido de difósforo al vacío.

Se pesaron $75 \pm 2 \mu \mathrm{g}$ de cada muestra en una barqueta de cuarzo, usando una balanza Mettler AT21 con una precisión de $1 \mu \mathrm{g}$.

La pirólisis fue efectuada en una cámara de pirólisis de marca Chemical Data System (CDS) 1000 acoplada a un cromatógrafo gaseoso modelo HP 5890 serie II, a través de una interface calentada a $270^{\circ} \mathrm{C}$. El pirolisado fue separado en una columna capilar DB1701 de $60 \mathrm{~m}$ con $0,25 \mathrm{~mm}$ de diámetro interno y $0,25 \mu \mathrm{m}$ de espesor. La temperatura de pirólisis fue de $650{ }^{\circ} \mathrm{C}$ durante $10 \mathrm{~s}$, con tiempo de subida de aproximadamente $800 \mathrm{~ms}$. Las condiciones del cromatógrafo gaseoso fueron las siguientes: inyector a $270^{\circ} \mathrm{C}$, detector a $270^{\circ} \mathrm{C}$ y una razón de separación (split) de $1 / 20$; programa de temperatura - $45^{\circ} \mathrm{C}$ durante $4 \mathrm{~min}$, seguido de una tasa de calentamiento de $4^{\circ} \mathrm{C} / \mathrm{min}$ hasta alcanzar $270{ }^{\circ} \mathrm{C}$, donde permaneció durante $6 \mathrm{~min}$. Los productos de la pirólisis fueron detectados por un detector de llama (Py-GC-FID).

La cuantificación de los productos de la pirólisis se basó en el cálculo de las áreas de sus picos, donde $\mathbf{h}$ representa la sumatoria de las áreas de los picos derivados de grupos p-hidroxifenilo, g la sumatoria de las áreas de los picos derivados de grupos guaiacilo, cP la sumatoria de las áreas de los picos de los marcadores de las pentosas, $\mathbf{c H}$ la sumatoria de las áreas de los picos de los marcadores de las hexosas y c la sumatoria de las áreas de los picos de los polisacáridos no atribuibles ni a pentosas 
ni a hexosas (Rodrigues et al., 1999). La información que proporcionó la pirólisis analítica se obtuvo en forma de pirogramas a través de los cuales se realizaron los cálculos, utilizando el software HP GC ChemStation (1990 - 1996).

El contenido de lignina Klason se realizó según Schwanninger et al., 2002. La hidrólisis se inició con la adición de $5 \mathrm{~mL}$ de ácido sulfúrico al $72 \%$ a $333 \mathrm{mg}$ de madera molida, libre de extractivos, durante dos horas en un baño de agua a $20^{\circ} \mathrm{C}$. A continuación, siguió una segunda hidrólisis diluyendo el ácido a $3 \%$ y colocando la mezcla en una autoclave a $120^{\circ} \mathrm{C}$ durante 1 hora. El residuo insoluble en ácido, filtrado en filtro G3 correspondió a la lignina Klason.

Fue utilizado el software STATISTICA 6.0. para el análisis estadístico de los resultados.

\section{RESULTADOS Y DISCUSION}

En la figura 3 se muestra un pirograma típico de los que fueron obtenidos para la especie Pinus caribaea var caribaea donde están señalizados los picos más importantes con la información que ellos ofrecen. Todos los pirogramas obtenidos presentan los mismos picos, varía únicamente la proporción relativa entre ellos.

Los resultados derivados del análisis de todos los pirogramas se tradujo en los cálculos de los contenidos de los grupos de compuestos más importantes como se explicó anteriormente. Se obtuvieron pirogramas semejantes a los obtenidos para otras especies de coníferas (Alves, 2004). El contenido de lignina fue calculado como el cociente entre la sumatoria de las áreas de los productos $\mathbf{h}$ y $\mathbf{g}$ y la sumatoria de las áreas de todos los productos identificados $(\mathbf{c}+\mathbf{c P}+\mathbf{c H}+\mathbf{g}+\mathbf{h})$.

Los productos eluidos en los primeros 29 minutos del pirograma son moléculas pequeñas de origen polisacárido, como el formaldehído, el acetaldehído, el propanal, la propanona y el ácido acético. A partir de ese momento, el pirograma es dominado por productos derivados de la lignina, siendo el primer componente el fenol $(45, \mathrm{~h})$, un derivado del hidroxifenilpropano. Los productos eluidos en los últimos 10 minutos tienen origen guayacílico, con excepción de dos, la levoglucosana $(96, \mathrm{cH})(\approx 49$ min.) originaria de la celulosa, y un azúcar anhidro $(51, \mathrm{c})(\approx 52 \mathrm{~min}$.). Es de notar que no fueron detectados productos de origen siringuílico.

Los contenidos de lignina así determinados se muestran en la tabla 1 . Al análisis estadístico, no existen diferencias significativas entre los contenidos de lignina entre las localidades, determinados por pirólisis analítica (tabla 2). 


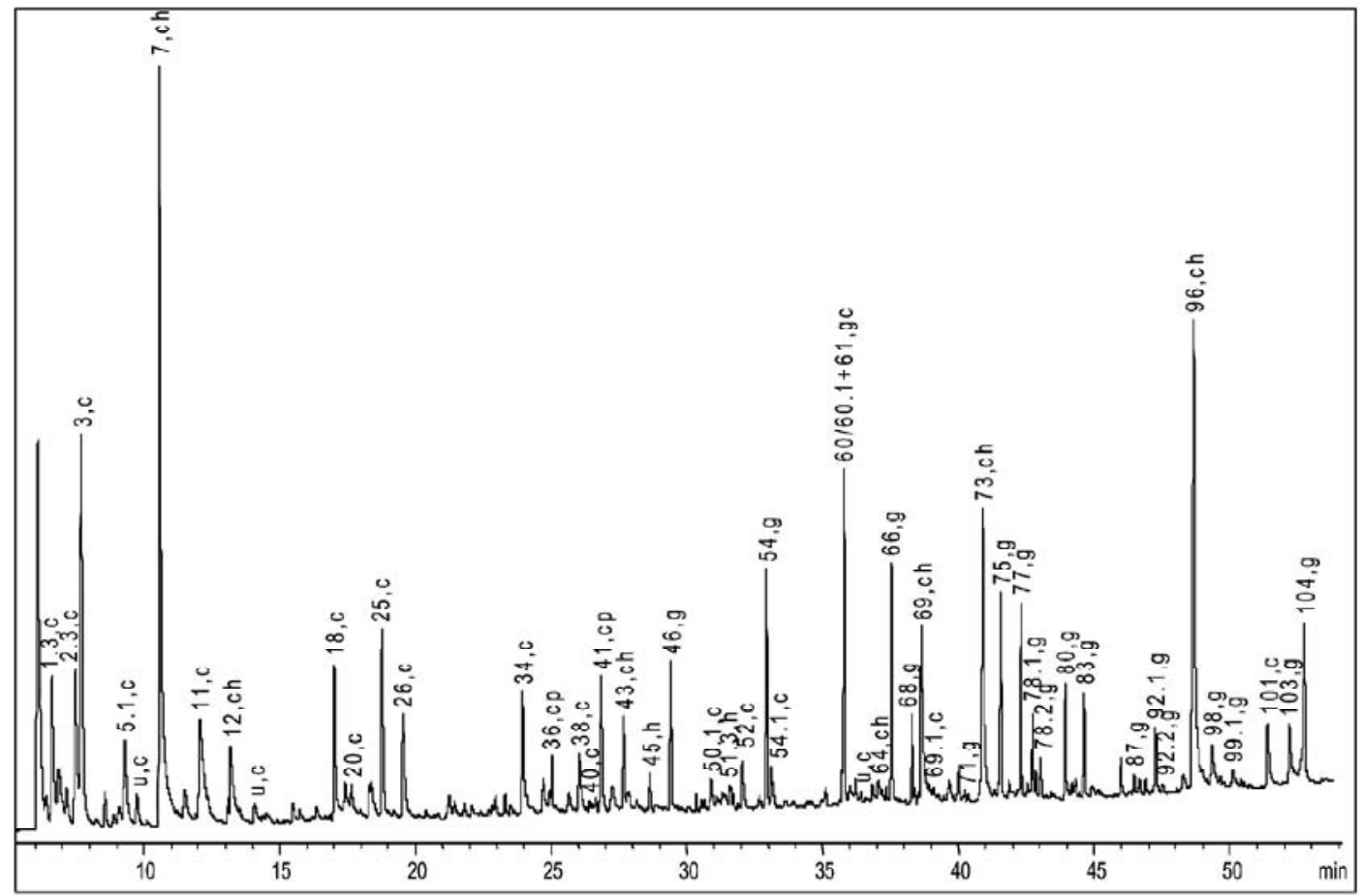

Figura 3. Pirograma tipo de madera de Pinus caribaea var caribaea

Con el objetivo de comparar los resultados del contenido de lignina obtenidos por pirólisis analítica se utilizó como referencia el método Klason. Se determinó el contenido de lignina a las 15 muestras representativas de las localidades y adicionalmente a 8 de las correspondientes a las diferentes alturas de la localidad de Malas aguas. En la tabla 1 se comparan los valores del contenido de lignina obtenidos mediante la pirólisis analítica con los obtenidos por el método tradicional (lignina Klason) para las tres localidades.

Tabla 1. Comparación del contenido de lignina por Klason y por pirólisis analítica

\begin{tabular}{c|ccc|ccc}
\hline & \multicolumn{3}{|c|}{ Lignina Klason } & \multicolumn{3}{c}{ Lignina - pirólisis } \\
\hline & Cajálbana & Marbajita & $\begin{array}{c}\text { Malas } \\
\text { aguas }\end{array}$ & Cajálbana & Marbajita & $\begin{array}{c}\text { Malas } \\
\text { aguas }\end{array}$ \\
\hline $\bar{x}$ & 26,6 & 26,4 & 28,6 & 24,9 & 24,8 & 25,7 \\
$\mathrm{SD}$ & 0,9 & 1,4 & 2,0 & 0,8 & 1,5 & 1,3 \\
$\mathrm{CV} \%$ & 3,5 & 5,2 & 7,2 & 3,4 & 6,1 & 5,0 \\
Mínimo & 24,4 & 25,4 & 25,8 & 22,5 & 23,6 & 23,5 \\
Máximo & 27,6 & 28,0 & 31,4 & 26,3 & 25,8 & 26,8 \\
\hline
\end{tabular}


Tabla 2. Análisis de varianza para contenido de lignina

\begin{tabular}{c|ccccc}
\hline $\begin{array}{c}\text { Origen de la } \\
\text { variación }\end{array}$ & $\begin{array}{c}\text { Grados de } \\
\text { libertad }\end{array}$ & $\begin{array}{c}\text { Suma de } \\
\text { cuadrados }\end{array}$ & $\begin{array}{c}\text { Media de la suma } \\
\text { de los cuadrados }\end{array}$ & F & P \\
\hline Entre localidades & 2 & 0,73 & 0,36 & 0,23 & 0,80 \\
Residual & 12 & 19,0 & 1,58 & & \\
\hline
\end{tabular}

(Diferencias significativas $\mathrm{p}<0,05$ )

En la figura 4 se presenta la correlación entre el porcentaje de lignina Klason y la determinada por pirólisis analítica para 23 muestras por ser el número máximo de muestras a las cuales se les realizó la determinación por Klason. Se obtuvo una correlación aceptable $\left(\mathrm{R}^{2}=0,765\right)$ lo cual permite estimar el contenido de lignina Klason en madera molida de Pinus caribaea var caribaea usando este modelo de regresión simple.

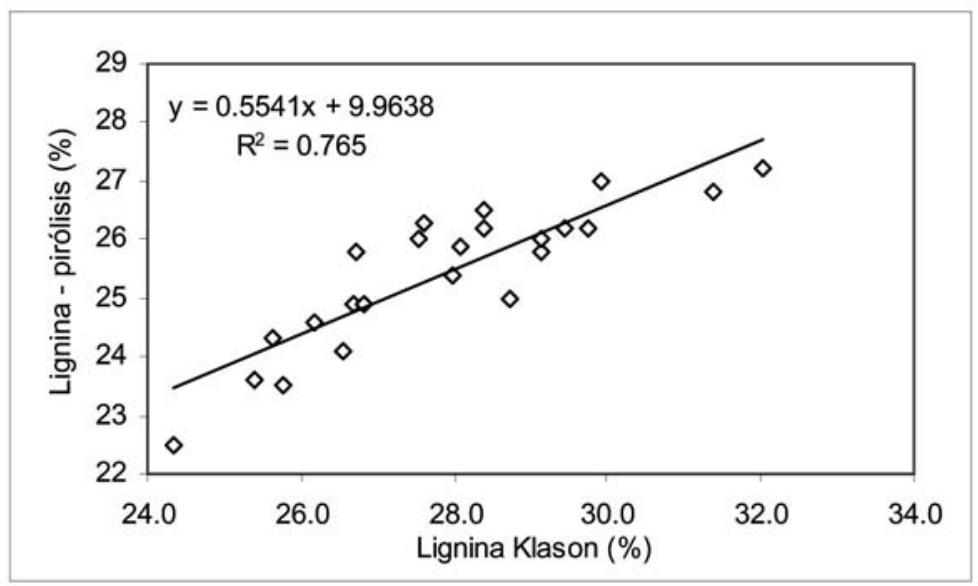

Figura 4. Correlación entre lignina Klason y lignina- pirólisis

En la tabla 3 se muestran los resultados obtenidos sobre la calidad de la lignina, expresada por la relación H/G, los cuales se obtuvieron del análisis de los pirogramas para las tres localidades. Las muestras estudiadas presentan un valor promedio de la relación H/G de 0,050, cercano al encontrado para Picea abies e inferior al reportado para Pinus taeda $(0,140)$ (Fengel y Wegener, 1984) y para Pinus pinaster (0,061) (Alves, 2004).

Estos autores han verificado que las muestras con contenido de lignina Klason y relaciones H/G más elevadas, correspondían a muestras que presentaban leño de reacción; cabe destacar que las muestras de madera de Pinus caribaea utilizadas en este estudio no se encontró presencia de leño de reacción. El leño de reacción de las resinosas, además de presentar mayor contenido de lignina que el leño normal, presenta mayores cantidades $(70 \%)$ de unidades de p-hidroxifenilo $(\mathrm{H})$, en relación al área total de los productos de la lignina (Sarkanen, 1971; Fengel y Wegener, 1984). 
Tabla 3. Variación de la relación H/G por localidades

\begin{tabular}{c|c|c|c}
\hline Localidad & \multicolumn{3}{|c}{ Relación H/G } \\
\hline & $\bar{x}$ & SD & CV \\
Cajálbana & 0,047 & 0,003 & 6,2 \\
Marbajita & 0,052 & 0,004 & 7,8 \\
Malas Aguas & 0,053 & 0,008 & 14,4 \\
\hline
\end{tabular}

Por el análisis estadístico no se observan diferencias significativas para la relación H/G entre las localidades como se muestra en la tabla 4.

Tabla 4. Análisis de varianza para la razón H/G

\begin{tabular}{c|ccccc}
\hline $\begin{array}{c}\text { Origen de la } \\
\text { variación }\end{array}$ & $\begin{array}{c}\text { Grados de } \\
\text { libertad }\end{array}$ & $\begin{array}{c}\text { Suma de } \\
\text { cuadrados }\end{array}$ & $\begin{array}{c}\text { Media de la suma } \\
\text { de los cuadrados }\end{array}$ & F & P \\
\hline Entre localidades & 2 & 0,0001 & 0,000056 & & \\
Residual & 12 & 0,0004 & 0,000031 & 1,8 & 0,21 \\
\hline
\end{tabular}

(Diferencias significativas $\mathrm{p}<0,05$ )

En la figura 5 se comparan mediante diagrama de máximos y mínimos, el contenido de la relación H/G determinado en madera de Pinus caribaea var caribaea con los resultados obtenidos para otras especies de coníferas por Alves (2004).

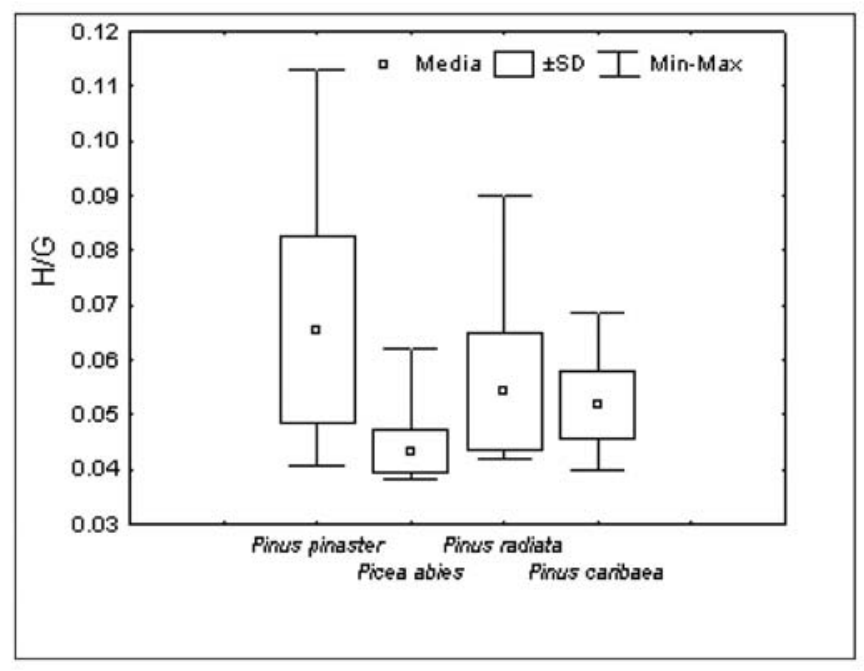

Figura 5. Comparación de la relación $\mathrm{H} / \mathrm{G}$ de madera de $P$. Caribaea var caribaea con la de otras pináceas. 


\section{CONCLUSIONES}

- En el trabajo realizado se aplicó la pirólisis analítica para la determinación del contenido y la composición de la lignina en madera de Pinus caribaea var caribaea procedentes de tres localidades de la provincia de Pinar del Río y no se apreciaron diferencias significativas entre las localidades para las magnitudes estudiadas.

- Este estudio reveló que la pirólisis analítica puede usarse para evaluar el contenido de lignina en madera de Pinus caribaea var caribaea con una precisión comparable al método de referencia (método Klason).

- La pirólisis analítica, a pesar de ser un método fácil de ejecutar, es muy sensible a pequeñas alteraciones del método experimental a aplicar. Este estudio constituye un primer acercamiento a una técnica muy útil para ensayos de grandes cantidades de muestra y especialmente para la industria de pulpa para papel, donde la relación $\mathrm{H} / \mathrm{G}$ puede significar un indicador en el proceso productivo en cuanto a la tasa de deslignificación.

\section{NOTA}

* Investigación realizada en: Centro de Estudio de Ingeniería Forestal. Instituto Superior de Agronomía. Universidad Técnica de Lisboa. Portugal.

\section{BIBLIOGRAFIA}

Alves, A. 2004. Caracterização da Composição Química da Madeira de pinheiro bravo (Pinus pinasyter Aitan) por pirólise analítica. Dissertação de Mestrado (Tesis de Magister), Universidade Técnica de Lisboa, Portugal.

Alves, A; Schwanninger, M.; Pereira , H.; Rodriguez, J. 2006. Calibration of NIR to assess lignin composition (H/G ratio) in maritime pine wood using analytical pyrolysis as the reference method. Holzforschung 60: 29-31.

Fengel, D. ; Wegener, G. 1984. Wood: chemistry, ultrastructure, reactions. Walter de Gruyter \& Co., Berlin.

Galletti, G. 1995. Analytical pyrolysis of lignocellulose, pulp and paper biotechnology. Curso Comett II ca 8490, FORBITEC.

Kuroda, K.; Izumi, A.; Mazumder, B.B.; Ohtani, Y.; Sameshima, K. 2002. Characterization of kenaf (Hibiscus cannabinus) lignin by pyrolysis-gas-chromatography-mass spectrometry in the presence of tetramethylamonium hydroxide. Journal of Analytical and Applied Pyrolysis 64: 453-463.

Meier, D.; Faix, O. 1992. Pyrolysis-gas Chromatography-mass Spectrometry of lignin. In: Methods in Lignin Chemistry. S. Lin y C. Dance (Ed.). Springer, Berlin.

Pereira, H.; Graça, J.; Rodrigues, J. 2003. Wood chemistry in relation to quality. In: Wood quality and its biological basis. John R. Barnett e George Jeronimidis (Ed.).

Rodrigues, J. 1998. Caracterização da Composição Química da Madeira de Eucalyptus globulus Labill. por espectroscopia de infravermelho e pirólise analítica. Dissertação de Doutoramento (Tesis de doctorado), Universidade Técnica de Lisboa, Portugal. 
Rodrigues, J.; Meier, D.; Pereira, H. 1999. Determination of tree to tree variation in syringyl:guaiacyl ratio of Eucalyptus globulus wood lignin by analytical pyrolysis. Journal of Analytical and Applied Pyrolysis 48: 121-128.

Sarkanen, K. 1971. Precursores and their polimerization. In: Lignins-Occurrence, Formation, Structure and Reactions. Eds. K. V. Sarkanen and C. H. Ludwig. Willey interscience. New York.

Schwanninger, M. ; Hinterstoisser, B. 2002. Klason lignin: Modifications to improve the precision of the standardized determination. Holzforschung 56 (2): 161-166. 
Departamento de Ingeniería en Maderas

$\approx \star$

Facultad de Ingeniería

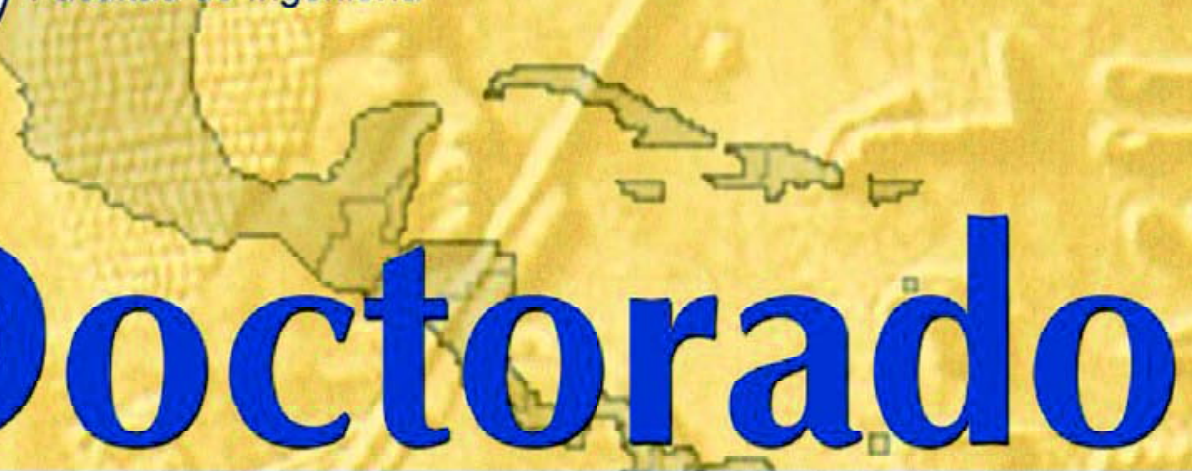

en Ciencias e lindustrias

de la Madera

LÍNEAS DE INVESTIGACIÓN

Propiedades de la Madera

Secado y Biotransformación de la Madera

Maderas Reconstituídas y Adhesivos

Producción, Gestión y Automatización

Estructuras y Productos de Ingeniería
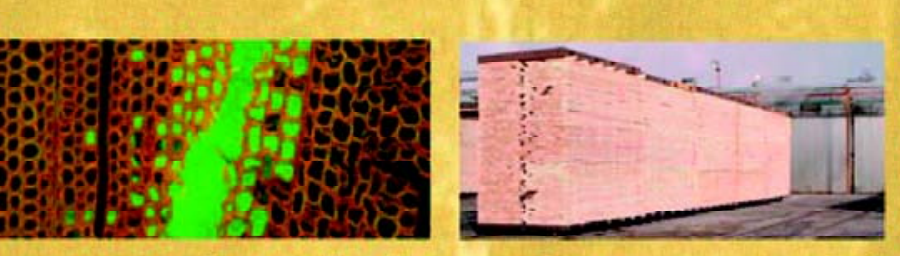

Chile

Concepción

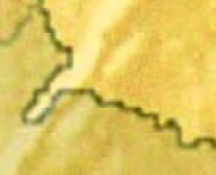

Postulaciones: Permanente, Inicio de Clases: Abril de cada año. MAGISTER en Ciencia y Tecnología de la Madera Contacto e información-adicional: 